\title{
Angiolipoma: A Rare Cause of Colonic Intususception
}

\author{
Dr. Badareesh Lakshminarayana, Dr. Annappa Kudva, Dr. Karthikeyan Selvam, \\ Dr. Vishal Ratkal \\ Kasturba Medical College Manipal \\ Department of Surgery KMC Manipal-576104
}

\begin{abstract}
:
Introduction: Intussusception in adult is an uncommon clinical entity. An intussusception involving left sided colon due to a benign tumor such as angiolipoma still more uncommon. Prompt pre-operative diagnosis using imaging technique helps in appropriate surgery. Case report: We present a 60years old patient who presented with features of intestinal obstruction who was evaluated with CECT and found to have multiple lipoma on left colon with diverticuli on right colon. On exploration he was found to have colocolic intusception at descending colon, one of the lipoma being the lead point along with right sided colonic diverticuli. He underwent segmental colectomy with transverse colon-sigmoid end to end anastomosis. Final histopathology was reported as multiple angiolipoma with diverticulosis. Conclusion: Pre-operative imaging techniques are of great value in identifying rarer aetiologies of colonic obstruction such as angiolipoma. Surgery forms the best treatment for those patients with symptomatic angiolipoma of colon.
\end{abstract}

Key words: Intussusception, Diverticuli, Angiolipoma

\section{Conflict of Interest Statement}

We, Badareesh L, Annappa Kudva, Karthikeyan Selvam and Vishal Rathkal declare that we don't have any conflict of interest in the present study in any form.

\section{Introduction:}

Angiolipomas are common benign tumours occurring at subcutaneous cellular tissue but are rare in gastrointestinal tract. Histologically, it is comprises of adipose tissue and proliferative blood vessels [1]. Many cases reported in the literature are diagnosed post-operatively. Proper usage of imaging technique helps in identifying the pathology and guides appropriate surgery.

\section{Case report:}

A 60 year old male presented with pain abdomen and distension of abdomen for 11 days and few episodes of vomiting. Patient was treated at local hospital for about a week conservatively. He was relieved of symptoms temporarily and had acute pain at presentation. He had a past history of surgery to the abdomen for unknown pathology. On examination, patient's vitals were stable. Per abdominal examination was grossly unremarkable except for diffuse tenderness and mild gaseous distension producing tympanic note all over abdomen. Bowel sounds were heard. Per rectal examination was unremarkable.

X-ray abdomen taken at casualty was non confirmative. Hence a CECT of abdomen was obtained. It showed multiple intraluminal fat density lesions suggestive of lipomas on left sided colon and multiple diverticuli on right sided colon. There was no evidence of any obstruction/intussusception or diverticulitis/perforation. As patient had severe pain and distension an explorative laparotomy was planned.

On laparotomy, he had a colo-colic intussusception at descending colon involving one of the lipoma as the leading point causing colonic obstruction [figure 1]. Other two lipomas were seen on proximal part of descending colon and splenic flexure respectively. As right sided colon had multiple diverticuli only (no evidence of diverticulitis/perforation), a segmental colectomy with transverse colon to sigmoid colon end to end anastomosis was done. Post op recovery was uneventful. Post op histopathology confirmed the presence of angiolipoma and diverticuli [figure 2].

\section{Discussion:}

In 1912, Bowen was the first one to describe about angiolipoma,[1]. Its clinical and pathological features which are different from lipoma were described in 1960 by Howard and Helwig,[2]. It's a well encapsulated tumour in young adults commonly seen in arms and trunk,[3, 4]. Submucosal lipoma is the 2nd most common benign colonic tumor after adenoma,[5]. Larger lipomas are likely to cause symptoms, such as bleeding, obstruction, and intussusception. 
Preoperative imaging allows the diagnosis of angiolipoma. On ultrasound, the texture varies from hypoechogenic to hyper-echogenic depending on the fat content of the tumour. Pattern on CT also depends on the composition of fat in the tumour. A tumour with low fat content shows numerous round density enhancements, $[6,7]$.

Operative intervention is indicated in all cases of adult intussusception,[8]. Urgent treatment should be given to those with intussusception, obstruction, or bleeding,[1]. As angiolipoma are benign tumours, when planned for surgery the colon resected should be kept to the minimum.

In the present case, we could pre-operatively locate multiple pathologies (angiolipoma \& diverticuli) but could not explain which one was the culprit for patient's symptoms. Intra-operatively, the presence of intussusception explained the reason for obstruction. The presence of multiple diverticuli in the right sided colon without any complications precluded us from performing an total colectomy and hence a segmental colectomy was performed.

\section{Conclusion:}

Pre-operative imaging techniques are of great value in identifying rarer aetiologies of colonic obstruction such as angiolipoma. Surgery forms the best treatment for those patients with symptomatic angiolipoma of colon.

\section{Reference:}

[1]. Chen YY, Soon MS. Preoperative diagnosis of colonic angiolipoma: A case report. World J Gastroenterol 2005; 11 (32): 50875089.

[2]. Howard WR, Helwig EB. Angiolipoma. Arch Dermatol 1960; 82: 924-931.

[3]. Ackermano LV, Rosai J. Tumors of adipose tissue. In: Rosai J, editor: Akerman's surgical pathology.8th ed Vol 12.St Louis: Mosby 1996: 2053-2060.

[4]. Enzinger FM, Weiss SW. Soft tissue tumors 3rd ed. Benign lipomatous tumors, St. Louis Mosby 1995: 390.

[5]. Ginzburg L, Weingarten M, Fischer MG. Submucous lipoma of the colon. Ann Surg 1958;148:767-72.

[6]. Pfeil SA, Weuver MG, Abdul- Karim FW et al. colonic lipomas: outcome of endoscopic removal. Gastrointest Endosc 1990; 36: 435-438.

[7]. Beatriz Molinares, Andrés Goldstein, Gabriel J Varela et al. Colonic Angiolipoma - A rare finding in the gastrointestinal tract. Case Report and review of literature. Radiology Case. 2012 Jun; 6(6):23-28.

[8]. Stephen P. Courtney, Nassif Ibrahim, Andrew J. Longstaff et al. Intussusception in the adult: clinical, radiological and histological features. Postgrad Med J (1992) 68, 449 - 452.

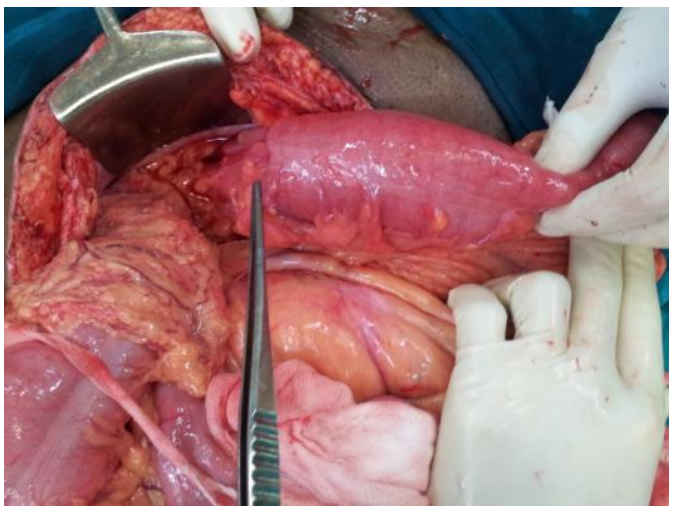

Figure 1- intra-op photo showing colo-colic intususception

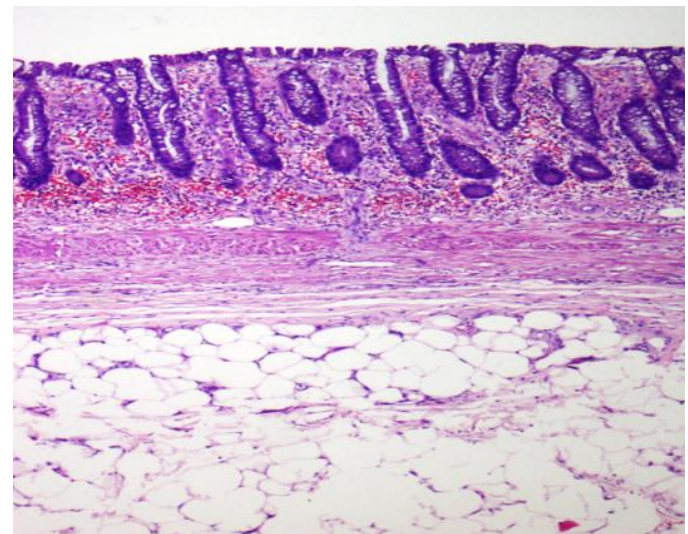

figure 2-HPE slide showing submucosal lipoma with extensive vasculature in lamina propria 